\title{
Fístula gastroesplénica por adenocarcinoma gástrico y peritonitis difusa
}

\section{Gastrosplenic fistula due to gastric adenocarcinoma and diffuse peritonitis}

\author{
Luis E. Terry Castellano ${ }^{1, a}$, Jessica Tatheana Matheus Saintupac ${ }^{2, b}$ \\ ${ }^{1}$ Servicio de Cirugía de Emergencias, Departamento de Cirugía General, Hospital Nacional Guillermo Almenara Irigoyen, EsSalud. Lima, Perú. \\ ${ }^{2}$ Servicio de Anatomía Patológica y Necropsias, Departamento de Patología, Hospital Nacional Guillermo Almenara Irigoyen, EsSalud. Lima, Perú. \\ ${ }^{a}$ Médico cirujano general, maestrando en epidemiologia. ORCID: https://orcid.org/0000-0001-7783-0182 \\ ${ }^{b}$ Médica patóloga, ORCID: https://orcid.org/0000-0001-9658-2065
}

An Fac med. 2021;82(2):146-9. / DOI: https://doi.org/10.15381/anales.v82i2.16907.

\begin{abstract}
Correspondencia:
Luis Terry Castellano

luisterryc@hotmail.com
\end{abstract}

Recibido: 15 de enero 2021

Aprobado: 17 de junio 2021

Publicación en línea: 5 de agosto 2021

Conflictos de interés: Los autores declaran no tener conflictos de interés.

Fuente de financiamiento:

Autofinanciado

Citar como: Terry L, Matheus J. Fistula gastroesplénica por adenocarcinoma gástrico y peritonitis difusa. An Fac med. 2021;82(2):146-9.DOl: https://doi. org/10.15381/anales.v82i2.16907

\section{Resumen}

Se reporta el primer caso en Perú de una fístula gastroesplénica (FGE) secundaria a adenocarcinoma gástrico penetrante al hilio esplénico que ocasionó infarto esplénico maligno y peritonitis difusa. Se discute la racionalidad del tratamiento quirúrgico. Varón de 84 años con peritonitis difusa secundario a un adenocarcinoma gástrico Borrmann III (BIII) fístulizado al bazo y en comunicación con la cavidad peritoneal. Se hallaron en la literatura cuatro casos de FGE asociadas a cáncer gástrico. La gastrectomía con intención curativa en un solo tiempo estaría indicada para casos con buena condición general y criterios de curabilidad. La gastrectomía en un segundo tiempo se reserva para casos con mala condición general y criterios de curabilidad. Las resecciones locales o paliativas estarían indicadas para los casos con mala condición general e incurabilidad.

Palabras clave: Fistula; Cáncer Gástrico; Bazo; Infarto del Bazo; Peritonitis; Perú (fuente: DeCS BIREME).

\section{Abstract}

The first case in Peru of a gastro-splenic fistula (GSF) secondary to gastric adenocarcinoma penetrating the splenic hilum, causing malignant splenic infarction and diffuse peritonitis, is reported. The rationale for surgical treatment is discussed. An 84-year-old man with diffuse peritonitis secondary to a Borrmann III (BIII) gastric adenocarcinoma fistulized to the spleen and in communication with the peritoneal cavity. Four cases of GSF associated with gastric cancer were found in the literature. The gastrectomy with curative intention in a single stage, would be indicated for cases with good general condition and criteria of curability. A second stage gastrectomy is reserved for cases with poor general condition and curability criteria. Local or palliative resections would be indicated for cases with poor general condition and incurability.

Keywords: Fistula; Stomach Neoplasms; Spleen; Splenic Infarction; Peritonitis; Peru (source: MeSH NLM). 


\section{INTRODUCCIÓN}

Biológicamente, el bazo es resistente a ser infiltrado o involucrado por neoplasias malignas de órganos vecinos o distantes. En autopsias de enfermos fallecidos por alguna neoplasia, entre el 0,6\% al $3 \%$ tienen metástasis al bazo ${ }^{(1,2)}$. La infiltración y perforación esplénica por un cáncer gástrico es rara; así, se han reportado 10 casos de 2564 (menos de 0,5\%).

Presentamos el primer caso publicado en el Perú, de una fístula gastroesplénica (FGE) secundaria a adenocarcinoma de cuerpo gástrico Borrmann III (BIII) infiltrante al hilio esplénico que ocasionó infarto, perforación, fístula y peritonitis difusa. Se revisa la literatura relacionada a cáncer gástrico complicado con FGE y su tratamiento.

\section{REPORTE DE CASO}

Paciente varón de 84 años, admitido en emergencia por sepsis abdominal y antecedente diagnóstico de adenocarcinoma gástrico poco diferenciado de curvatura mayor e infarto de miocardio, ambos 7 meses previos. Al examen físico: PA: 110/80 mmHg, FC: 110/min, FR: 24/ min. $\mathrm{T}: 38^{\circ} \mathrm{C}$, IMC: $17,7 \mathrm{Kg} / \mathrm{m}^{2}$. Abdomen: signo de Blumberg +. Score de Glasgow: 15. Analítica: leucocitos: $6410 / \mathrm{mm}^{3}$, abastonados 18\%, hemoglobina: 9,4 g/ $\mathrm{dL}$, plaquetas: $264000 / \mathrm{mm}^{3}$, albúmina: $2,3 \mathrm{~g} / \mathrm{dL}$, creatinina: $0,7 \mathrm{mg} / \mathrm{dL}, \mathrm{pH}$ : 7,32, pCO2: 36,4 mmHg, Na: $135 \mathrm{mEq} / \mathrm{L}$, $\mathrm{K}: 3,2 \mathrm{mEq} / \mathrm{L}$, lactato: $1,5 \mathrm{mmol} / \mathrm{L}$. Index APACHE II (Acute Physiology And Chronic Health Evaluation II): 15. Index SOFA (Sequential Organ Failure Assessment Score): 1. Index de Peritonitis Mannheim (IPM): 29 (mortalidad 70-85\%).

La tomografía abdominal con contraste mostró una imagen heterogénea, irregular e hipocaptante del hilio esplénico (necrosis). Burbujas de aire (aero-esplenia) e hipodensidad del parénquima en relación a absceso. Figura 1.

Con el diagnostico de abdomen agudo quirúrgico, se realizó laparotomía exploradora donde se evidenció pus libre en cavidad de aproximadamente 1000 cc. Esplenomegalia. Estómago: úlcera gás-

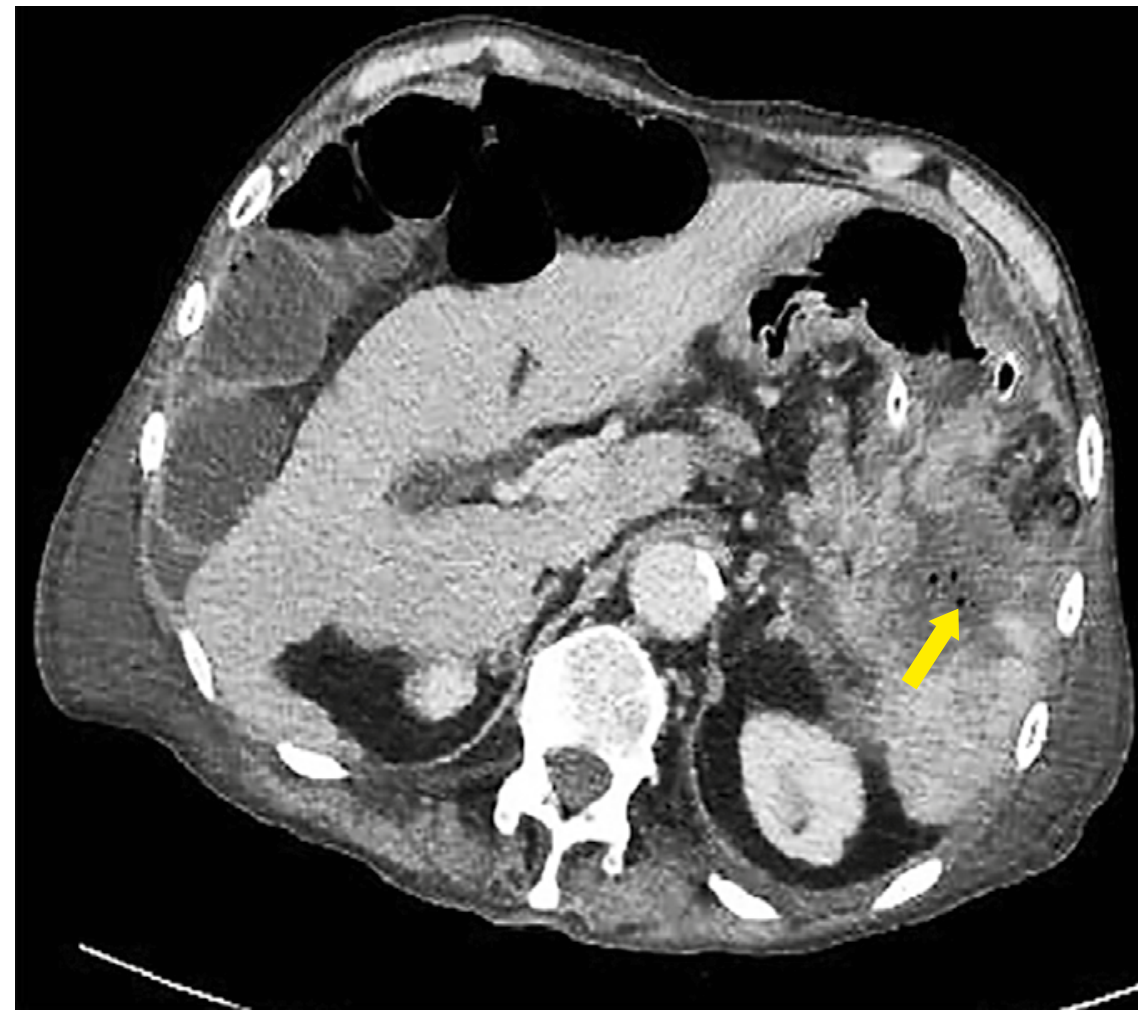

Figura 1. Tomografía con contraste que muestra imagen heterogénea, irregular e hipocaptante del hilio esplénico (necrosis). Burbujas de aire (aero-esplenia) e hipodensidad del parénquima en relación a absceso (flecha).

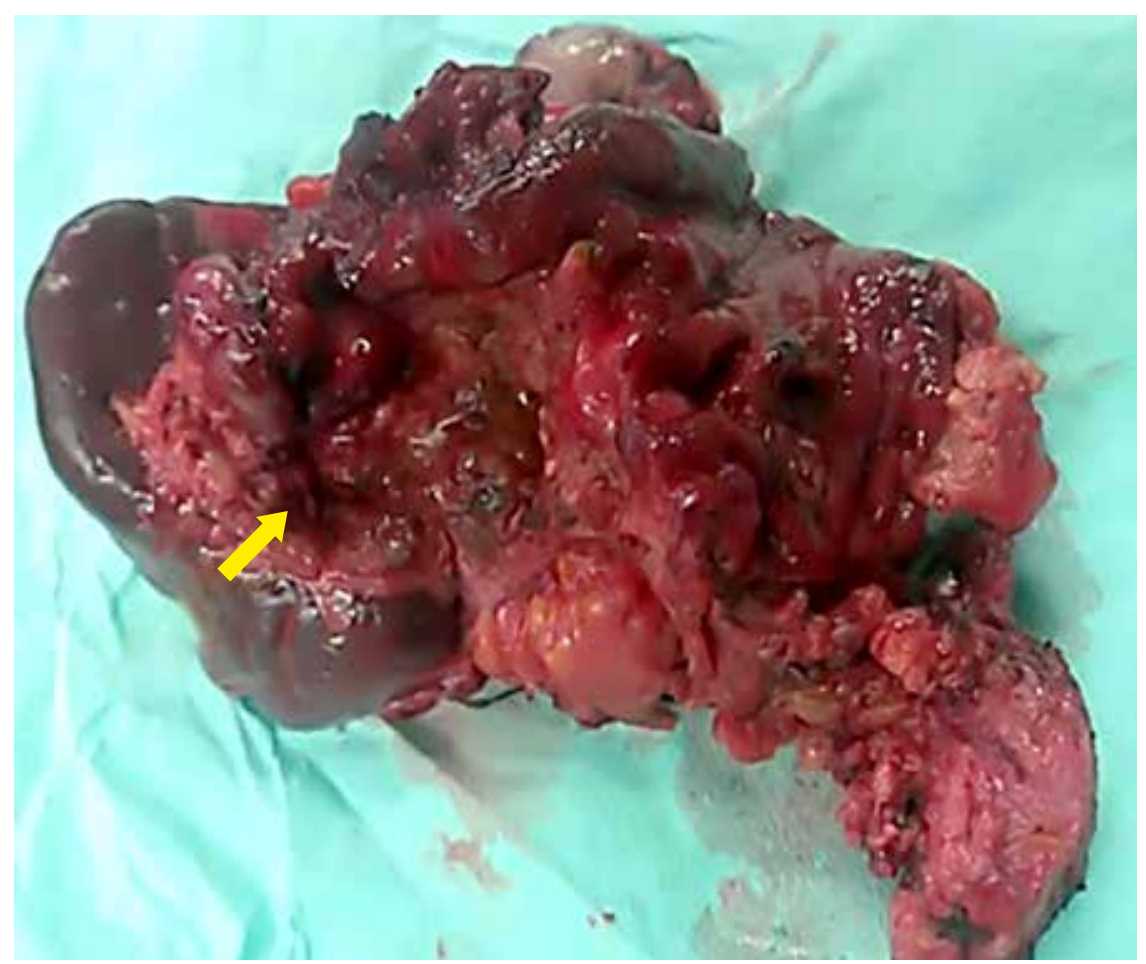

Figura 2. Vista de la cara interna o gástrica del bazo. Se aprecia úlcera gigante del cuerpo del estómago, de $70 \mathrm{~mm}$ de diámetro, irregular e indurada (flecha). Infiltra el hilio esplénico, atraviesa el parénquima hasta su cara diafragmática. 


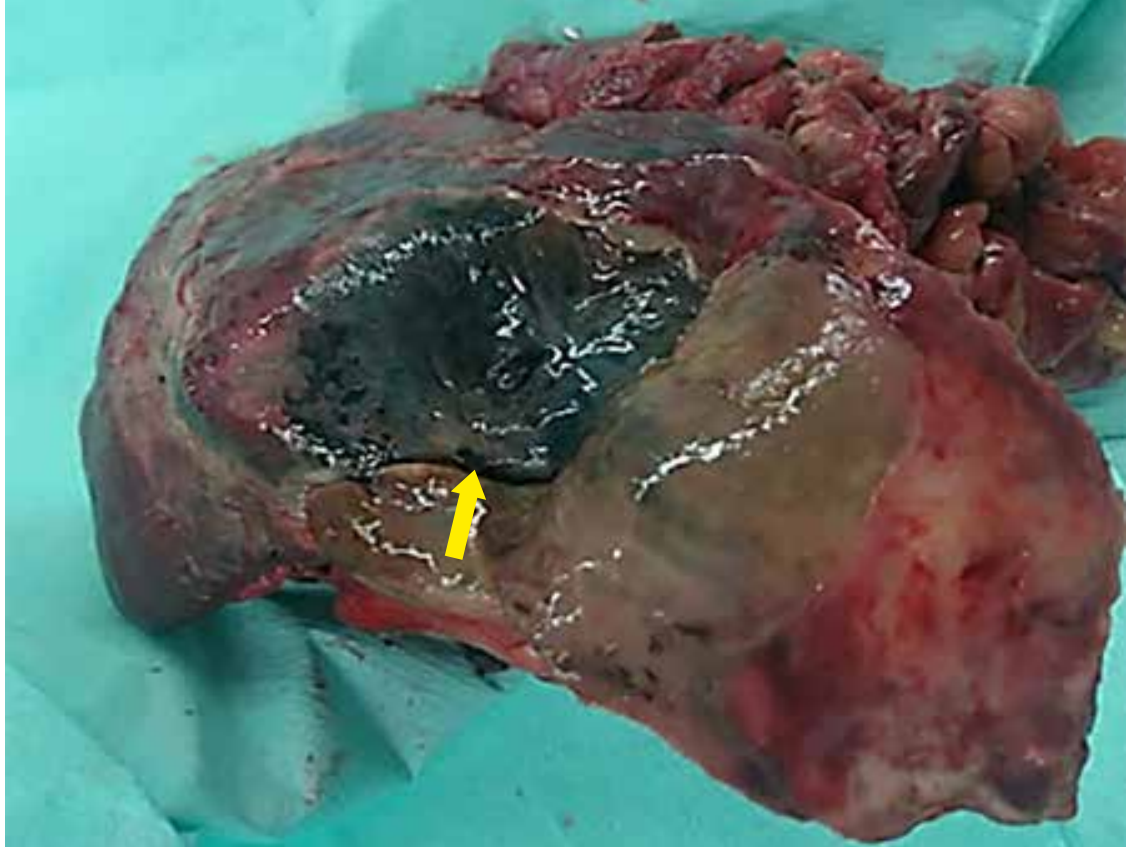

Figura 3. Vista de la cara diafragmática del bazo. Infarto esplénico segmentario (flecha) por trombosis maligna de su hilio, fistulizando estómago y cavidad peritoneal.

trica en curvatura mayor del cuerpo gástrico, de bordes indurados, de $7 \mathrm{~cm}$ de diámetro, penetrante al bazo a través de su hilio hasta necrosar la cápsula espléni-

ca en su cara diafragmática, tal como se muestran en las figuras 2 y 3 . Se realizó esplenectomía total, gastrectomía subtotal parcial longitudinal (resección R2,

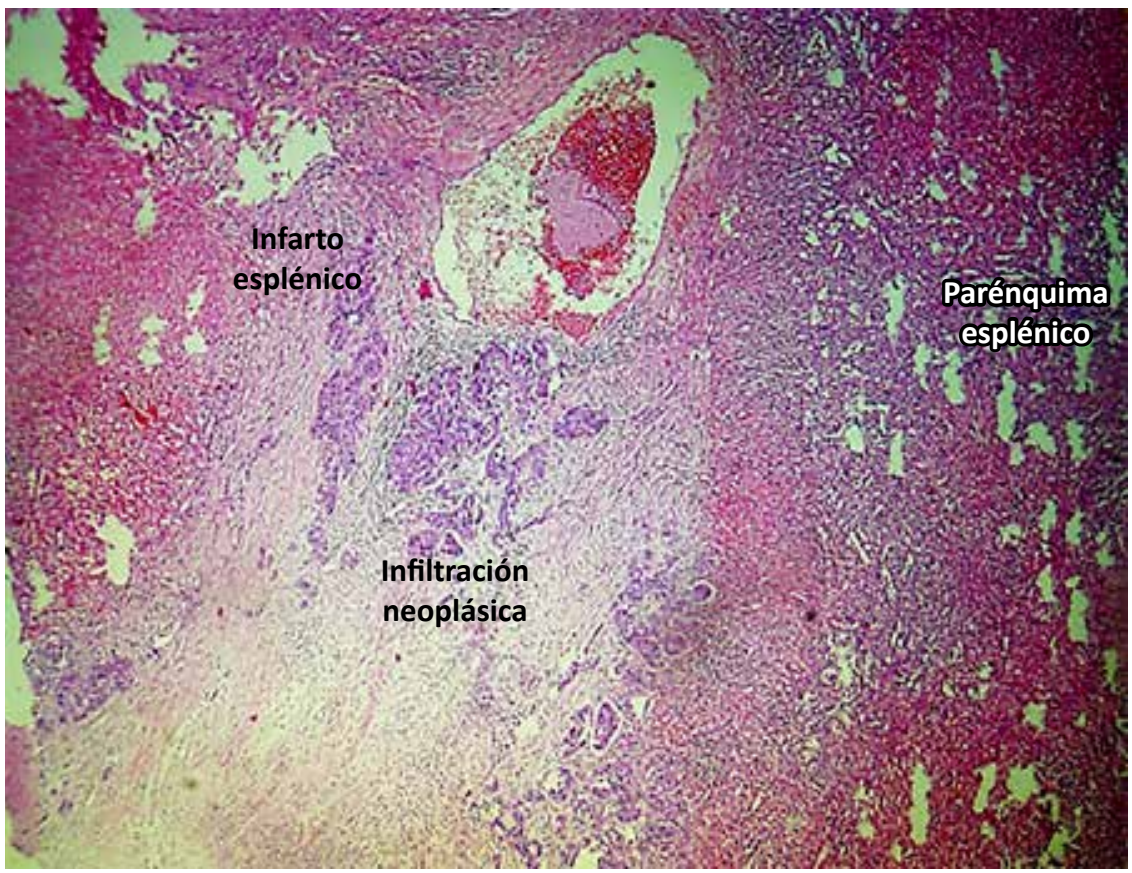

Figura 4. Microscopía del hilio esplénico.Infiltración neoplásica del hilio esplénico que ocasiona trombosis de los vasos de la pulpa roja e infarto consecuente. Carcinoma mixto, patrón tubular sólido y células en anillo de sello (adenocarcinoma poco diferenciado G3). Hematoxilina- eosina, 20x. linfadenectomía D0), yeyunostomía para alimentación, lavado de cavidad y drenaje. Pobre condición del paciente para cirugía radical.

El estudio anatomopatológico mostró infiltración neoplásica del hilio esplénico que ocasiona trombosis de los vasos de la pulpa roja e infarto consecuente así como carcinoma mixto, patrón tubular sólido y células en anillo de sello (adenocarcinoma poco diferenciado G3), tal como se muestra en la figura 4.

En el seguimiento del paciente, falleció tres meses después en su domicilio.

\section{DISCUSIÓN}

La primera fístula gastroesplénica (FGE) fue descrita por De Scoville (3) en 1962, a partir de una imagen tomográfica caracterizada por esplenomegalia y aire en el parénquima esplénico (aeroesplenomegalia), que luego se confirmó correspondía a un linfosarcoma esplénico necrótico fistulado al estómago. La FGE es una rara complicación potencialmente fatal de varias condiciones patológicas, que incluyen linfoma gástrico o esplénico, adenocarcinoma gástrico, enfermedad de Crohn, úlcera gástrica benigna, absceso esplénico y trauma. De todos estos, los linfomas difusos, linfomas de células $B, y$ de células grandes, son las neoplasias más frecuentemente involucradas en la creación de FGE ${ }^{(3)}$.

Dong Hyeok y col. identificaron $27 \mathrm{ca}$ sos de FGE por linfomas, de edades promedio 50,6 \pm 16,8 años, donde los síntomas más frecuentes fueron hemorragia digestiva alta, baja de peso y fiebre ${ }^{(4)}$. La tomografía axial computarizada con contraste fue el estudio de elección en los estudios publicados ${ }^{(5)}$.

¿Practicar gastrectomía subtotal longitudinal, resección R2, linfadenectomía DO, esplenectomía total, en este paciente con peritonitis difusa y sepsis grave fue razonable?. Gertsch ${ }^{(6)}$ reportó los resultados en 34 casos de cánceres gástricos perforados (CGP). De ellos, 30 (88\%) fueron sometidos a gastrectomía de emergencia con una mortalidad de $16 \%$. Seis continuaron vivos a 42 meses de seguimiento. Lehnert ${ }^{(7)}$, de un total de 
1273 pacientes con cáncer gástrico (entre 1982-1999) encontró 23 (1,8\%) casos complicados con perforación; y de 161 casos de perforaciones gástricas en el mismo periodo de estudio, 23/161 (14\%) ocurrieron desde un lecho maligno. Dos pacientes fueron gastrectomizados con intención curativa en emergencia (onestage procedure), 1 (50\%) falleció en el postoperatorio. Seis pacientes fueron tratados conservadoramente en emergencia y luego electivamente gastrectomizados con fines curativos (two-stage procedure), no hubieron fallecidos. De 8 pacientes sometidos a gastrectomía con intención curativa, 4 (50\%) estuvieron vivos a 5 años de seguimiento. De los sometidos a cirugía paliativa, ninguno alcanzó 6 meses de sobrevida. De la publicación de Lehnert ${ }^{(7)}$ se puede concluir que tratar la perforación gástrica maligna con gastrectomía radical y D2 no es aconsejable en emergencia; tratar la sepsis es prioritario. Se planifica la gastrectomía radical en segunda etapa (two-stage procedure).

Adachi y col. ${ }^{(8)}$ analizaron los resultados de 155 pacientes japoneses con cáncer gástrico perforado (CGP) reportados 10 años previos. Hubieron 27 EC (estadio clínico) I (19\%), 16 EC II (12\%), 42 EC III (30\%), 55 EC IV (39\%). Se realizaron gastrectomías de emergencia en 128 (83\%) pacientes con una tasa de mortalidad global de $7 \%$ y supervivencia a 5 años de $40 \%$. La tasa de sobrevida a 5 años para el EC I fue 93\%, para el EC II 49\%, para el EC III 34\% y para el EC IV 7\%. Para el mismo periodo de estudio, la sobrevida a 5 años de pacientes sometidos a gastrectomía electiva por CG no perforado, fue: 7896\% para el EC I, 46-77\% para el EC II, 20-
51\% para el EC III, y $10-20 \%$ para el EC IV; la sobrevivencia en ambas poblaciones (cáncer gástrico perforado y no perforado) fueron estadísticamente semejantes. La perforación gástrica y contaminación peritoneal no necesariamente influyeron en los resultados; solo el estadio clínico fue relevante. Adachi ${ }^{(8)}$ consideró que los exitosos resultados son atribuibles a la radicalidad de las gastrectomías, ausencia de compromiso hepático y peritoneal, quimioterapia postoperatoria y lavado profuso de la cavidad peritoneal.

Roviello (9) reportó la experiencia con $10(0,39 \%)$ casos de cáncer gástrico perforado (CGP) de 2564 CG operados en tres centros oncológicos de Italia. Es destacable el orden que propone para el adecuado abordaje de CGP teniendo en consideración lo que denomina la condición general del enfermo. Esta condición general estaría compuesta por las variables: condición hemodinámica (presencia o ausencia de shock), gravedad de la peritonitis y las comorbilidades eventuales. El factor curabilidad de la neoplasia es también importante para diseñar la conducta quirúrgica. Así, se pueden presentar 4 escenarios en pacientes con patología probada de cáncer: 1) Buena condición general + curabilidad aceptable = gastrectomía D1, D2 o gastrectomía en segundo tiempo. 2) Buena condición general + incurabilidad = gastrectomía paliativa. 3) Mala condición general + curabilidad aceptable = gastrectomía en segundo tiempo. 4) Mala condición general + incurabilidad = cirugía local (cierre simple). Si no se tiene certeza de la existencia de neoplasia, la condición general sola determina el proceder quirúrgico. Perforación + buena condición general = cirugía resectiva. Perforación + mala condición general = cirugía local reparadora, cierre simple.

Según lo sugerido por Lehnert ${ }^{(7)}$ (paciente sin condiciones ni diagnóstico de cáncer) y Roviello ${ }^{(9)}$ (mala condición general e incurabilidad), en el paciente del presente reporte se realizó gastrectomía parcial + esplenectomía + R2 + D0, con criterio paliativo.

\section{REFERENCIAS BIBLIOGRÁFICAS}

1. Schon CA, Gorg C, Ramaswamy A, Barth PJ. Splenic metastases in a large unselected autopsy series. Pathology - Research and Practice. 2006; 202(5): 351-356. DOI: https://doi.org/10.1016/j.prp.2005.12.008

2. Lam KY, Tang V. Metastatic tumors to the spleen: a 25-year clinic pathologic study. Arch Pathol Lab Med. 2000 Apr; 124(4):526-30. DOI: 10.5858/2000-124-0526-MTTTS

3. De Scoville A, Bovy P, Demeester P. 'Aerosplenomegalie' radiologique par lymphosarcome splenique necrosant a double fistulation dans le tube digestif. Acta Gastroenterol Belg. 1967; 30:841-7.

4. Kang DH, Huh J, Lee JH, Jeong YK, Jeong Y. Gastrosplenic fistula occurring in lymphoma patients: Systematic review with a new case of extranodal NKTT-cell lymphoma. World J Gastroenterol. 2017 Sep 21; 23(35): 6491-6499. DOI: 10.3748/wjg.v23.i35.6491

5. Knaus WA, Draper EA, Wagner DP, Zimmerman JE. APACHE II: a severity of disease classification system. Crit Care Med. 1985 Oct; 13(10):818-29.

6. Gertsch P, Yip SKH, Chow LWC, Lauder IJ: Free perforation of gastric carcinoma. Arch Surg. 1995; 130: 177 -181. DOI: 10.1001/archsurg.1995.01430020067011

7. Lehnert T, Buhl K, Dueck M, Hinz U, Herfarth C. Twostage radical gastrectomy for perforated gastric cancer. European Journal of Surgical Oncology. 2000; 26 (8): 780-784. DOI: https://doi.org/10.1053/ejso.2000.1003

8. Adachi Y, Mori M, Maehara Y, Matsumata T, Okudaira $Y$, Sugimachi K. Surgical results of perforated gastric carcinoma: an analysis of 155 Japanese patients. Am J Gastroenterol. 1997; 92(3): 516-518.

9. Roviello F, Rossi S, Marrelli D, De Manzoni G, Pedrazzani C, Morgagni $\mathrm{P}$, et al. Perforated gastric carcinoma: a report of 10 cases and review of the literature. World Journal of Surgical Oncology. 2006; 4:19. DOI: https:// doi.org/10.1186/1477-7819-4-19 